\title{
Drag-Reducing Effect in Solutions of Very High Molecular Weight Polystyrene by the Rolling Ball Method
}

\author{
Kunio NaKamura, Shinichi Odajima, Kazuya Kizawa, \\ and Tsurutaro NAKAGAWA ${ }^{\dagger}$ \\ Department of Polymer Science, Faculty of Science, Hokkaido University, \\ Kita 10-jo, Nishi 8-chome, Kita-ku, Sapporo 060, Japan
}

(Received September 24, 1987)

KEY WORDS Drag Reduction / Polystyrene / Rolling Ball Apparatus /
Turbulent Flow /

The first published data on the frictionreducing effect of polymer solutions in turbulent pipe flow were presented by Toms. ${ }^{1}$ Hence, turbulent drag reduction is sometimes referred to as the Toms effect. Many efforts have been directed towards elucidating the relations between the molecular characteristics of polymer additives and their drag reducing efficiency, mainly in regard to pipe flow.

We studied previously the drag reduction properties of very high molecular weight polystyrene solutions by the falling ball method, ${ }^{2}$ and those of aqueous solutions of sodium polyacrylate by the rolling ball method, ${ }^{3}$ respectively. The drag reduction effect in the flow around the solid bodies can be of considerable practical importance. In recent years, there has been an increasing interest in using drag reducing additives as possible circulation aids in the blood flow. ${ }^{4}$ The purpose of the present work was to investigate the effects of polymer concentration and solvent on the drag reduction efficiency in polystyrene solutions.

\section{EXPERIMENTAL}

The polymer used was unfractionated ultra- high molecular weight polystyrene, a commercial product (BK-2500) of the Japan Synthetic Rubber Co., having $\bar{M}_{w}=1.8 \times 10^{7}$ and polydispersity, $\bar{M}_{w} / \bar{M}_{n}=1.20$. The preparation of sample solutions is described elsewhere. $^{2}$ The solvent used were benzene and ethylbenzene.

The viscosity of the solutions was measured by a Ubbelohde viscometer. The value of the intrinsic viscosity and Huggins' constant for BK-2500 in benzene at $25^{\circ} \mathrm{C}$ was $21.3 \mathrm{dlg}^{-1}$ and 0.36 . The value of the intrinsic viscosity and Huggins' constant for BK-2500 in ethylbenzene at $25^{\circ} \mathrm{C}$ was $15.1 \mathrm{dl} \mathrm{g}^{-1}$ and 0.59 . The viscometric study of dilute solutions of BK2500 in various solvents was reported by Einaga et al. ${ }^{5}$

Drag reduction experiments were conducted with BK-2500 solutions ranging in concentrations from 0.001 to $0.01 \mathrm{~g} \mathrm{dl}^{-1}$ at $25^{\circ} \mathrm{C}$. The drag reduction properties of polymer solutions were measured by an inclined tube and rolling ball system.

The apparatus was designed so that observations of the ball velocity could be made over a range of angles from $15^{\circ}$ to $75^{\circ}$ from the horizontal. A $1.578 \mathrm{~cm}$ diameter glass tube and

\footnotetext{
${ }^{\dagger}$ Present address: Sapporo Gakuin University, 11, Bunkyodai, Ebetsu 069, Japan.
} 
steel balls with $1.4,1.2 \mathrm{~cm}$ in diameter were used in the experiments. The measuring technique is described elsewhere. ${ }^{4}$

We note that in this apparatus the ball falls, sliding on the wall of the inclined tube filled with a sample fluid, when the slope of tube exceeds a certain angle. In all such cases the results were discarded. A detailed discussion on the movement of the ball for aqueous solutions of gelation was given by Nagahora $e t$ al. ${ }^{6}$

According to Hubbard and Brown, ${ }^{7}$ the Reynolds number $R e$ is expressed by the equation:

$$
R e=\frac{l \rho u}{\eta}
$$

$l$ is the equivalent diameter which is equal to $(D-d)$ where $D$ is the tube diameter and $d$ the ball diameter. $u$ is the average fluid velocity related to the ball velocity $V$ by the equation,

$$
\frac{u}{V}=\frac{d^{2}}{D^{2}-d^{2}}
$$

$\rho$ is the density of fluid, and $\eta$ the viscosity of fluid.

The driving force $F$ on the ball is represented by the equation:

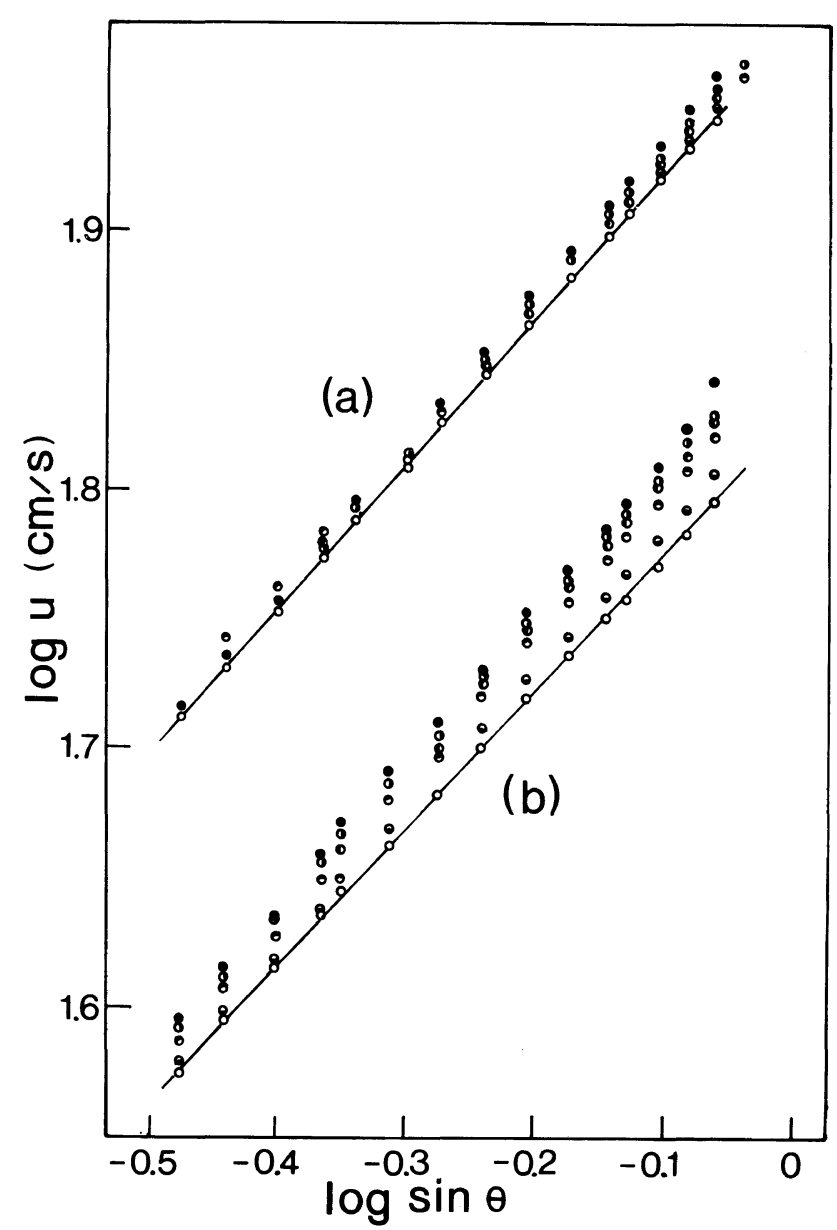

Figure 1. $\log u$ plotted against $\log \sin \theta$ for benzene and various BK-2500 solutions in benzene at $25^{\circ} \mathrm{C}$ : $\bigcirc$, benzene; $\ominus, 0.00102 ; \ominus, 0.00204 ; \bigcirc, 0.00408 ; \bigcirc, 0.00816 ; 0,0.0102 \mathrm{~g} \mathrm{dl}^{-1}$. (a) $l=0.178 \mathrm{~cm}$ and (b) $l=$ $0.378 \mathrm{~cm}$. 


$$
F=\frac{5}{7} \cdot \frac{1}{6} \pi d^{3}\left(\rho_{\mathrm{s}}-\rho\right) g \sin \theta
$$

where the coefficient $5 / 7$ is the fraction of the effective force of gravity $g$ that causes translational motion of the rolling ball and $\rho_{\mathrm{s}}$ is the density of the ball. In steady rolling motion, the driving force must be equal to the resisting force of the fluid.

\section{RESULTS AND DISCUSSION}

Figure 1 illustrates the $\theta$ dependence of $u$. It can be seen that in the higher range of $\sin \theta$ studied, the $u$ for the BK-2500 solutions is larger than that for the pure solvent indicating the drag reduction to occur by the addition of polystyrene.

The percent drag reduction $(D R)$ of polymer solutions at a constant Reynolds number of solvent $R e, s$ is defined by the following equation:

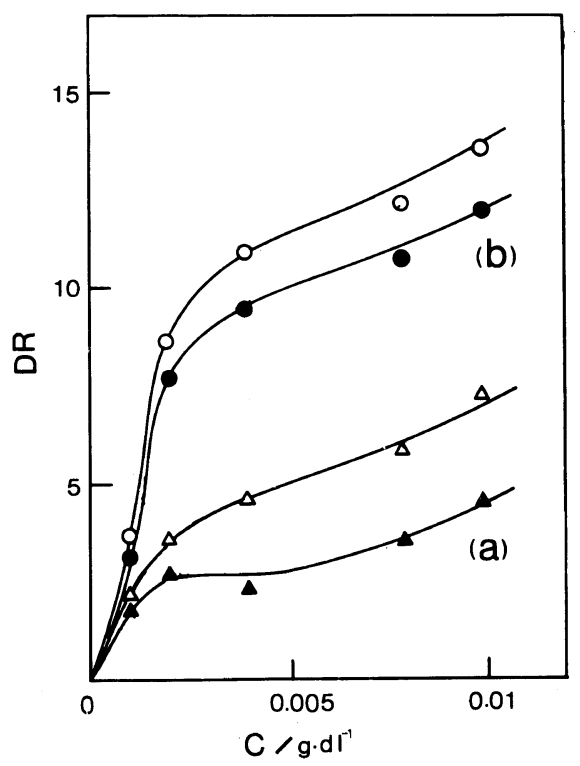

Figure 2. The drag reduction $D R$ plotted against the polymer concentration $c$ for the BK-2500 solutions in benzene at different $R e, s$. (a): $l=0.178 \mathrm{~cm} ; \triangle, R e, s=$ $1900 ; \Delta, 1700$. (b): $l=0.378 \mathrm{~cm} ; \bigcirc, R e, s=2900$; 2500 .

$$
D R=\frac{F_{\mathrm{s}}-F_{\mathrm{p}}}{F_{\mathrm{s}}} \times 100 .
$$

where $F_{\mathrm{s}}$ is the resisting force for the solvent as the standard, and $F_{\mathrm{p}}$ is that for the solution.

Figure 2 shows the percent drag reduction for BK-2500 solutions in benzene as a function of polymer concentration $c$ at $25^{\circ} \mathrm{C}$. It can be seen that $D R$ increases with increasing concentration and $R e, s$. A similar $D R$ curve was obtained in a previous study on $D R$ due to the same polystyrene solution in benzene by the falling ball method. ${ }^{2}$

Figure 3 shows the $c$ dependence of $D R$ for BK -2500 solutions in ethylbenzene at $25^{\circ} \mathrm{C}$. In this case, $D R$ initially increases, reaches a maximum value and then follows a plateau or decreases.

This $c$ dependence of $D R$ is a typical example of concentration effect. That is, drag reduction initially increases with concentration because of additional drag-reducing mol-

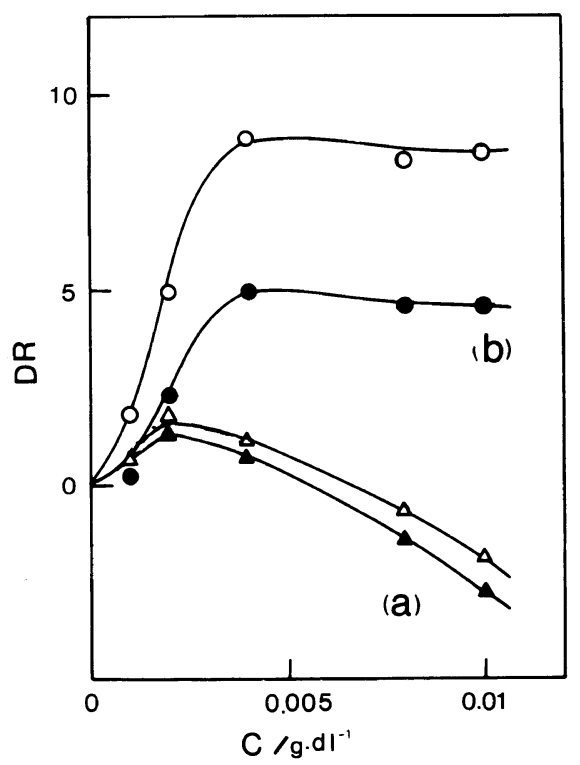

Figure 3. The drag reduction $D R$ plotted against the polymer concentration $c$ for the BK-2500 solutions in ethylbenzene at different $R e, s$. (a): $l=0.178 \mathrm{~cm} ; \triangle$, $R e, s=1900 ; \Delta, 1700$. (b): $l=0.378 \mathrm{~cm} ; \bigcirc, R e, s=2900$; 2500 . 
ecules. As the concentration is further increased, however, the solution viscosity also increases. This leads to an increase in drag. Eventually, the increased viscosity becomes the dominant factor, and the drag reducing efficiency reaches a maximum called the "effective plateau" and decreases.

The most striking feature of the drag reduction phenomenon is the low concentration involved in causing a substancial effect. A more effective agent may be one which achieves a required value of $D R$ at a lower concentration. The concentration to achieve $5 \% D R$ at $R e, s=2900$ for the BK-2500benzene system and for the BK-2500ethylbenzene system is $0.0012 \mathrm{~g} \mathrm{dl}^{-1}$ and $0.0020 \mathrm{~g} \mathrm{dl}^{-1}$, respectively. This suggests that BK-2500 is more effective in benzene than in ethylbenzene.

In Figure 4, the effects of $D R$ and reduced viscosity on the composition of the solvents of benzene and ethylbenzene of $0.0040 \mathrm{~g} \mathrm{dl}^{-1}$ BK-2500 solution at $25^{\circ} \mathrm{C}$ is shown. It can be seen that $D R$ decreases with the volume fraction of ethylbenzene $X_{2}$ in the mixed solvents

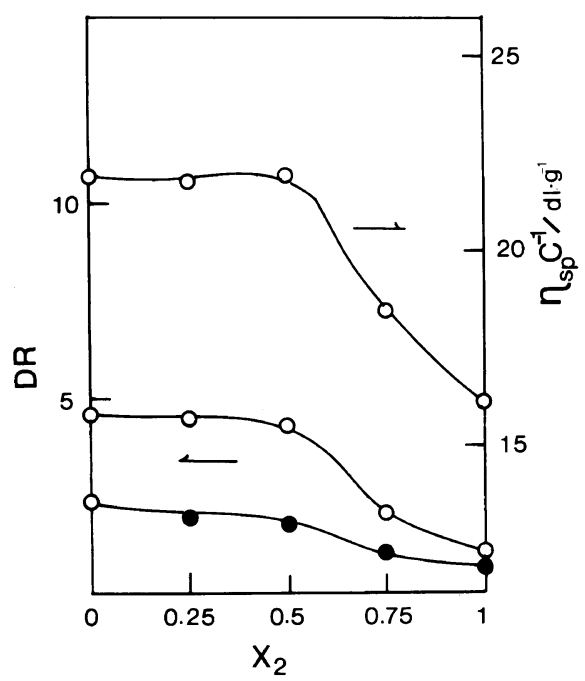

Figure 4. The drag reduction $D R$ and the reduced viscosity $\eta_{\mathrm{sp}} / c$ plotted against the volume fraction of ethylbenzene $X_{2}$ of the mixed solvents of benzene and ethylbenzene. The polymer concentration is $0.004 \mathrm{~g} \mathrm{dl}^{-1}$. $l=0.178 \mathrm{~cm} ; \bigcirc, \operatorname{Re}, s=1900 ; \bigcirc, 1700$. of benzene and ethylbenzene, and change in the reduced viscosity parallels the corresponding change in $D R$. It follows from this result that polystyrene acts as an effective reducer in a good solvent. These findings are in accordance with the results obtained for aqueous polyoxyethylene solutions in pipe flow experiments. ${ }^{8,9}$

$\mathrm{Kohn}^{10}$ proposed an energy storage theory for the drag reduction due to polymer solutions. The kinetic energy is dissipated as a result of turbulent mixing of fluid particles. However, when polymer molecules are present in the fluid, they respond to local stress by a conformational change and absorb and store energy in a deformed state. Our finding concerning the solvent effect on $D R$ may be interpreted as follows. The polymer chain is so expanded in good solvent that it is more easily deformable and more effective for absorbing the kinetic energy of the fluid.

Finally, the drag reduction determined by the rolling ball apparatus could be used to predict the effectiveness of an additive as a drag reducer. It is probable that drag on spheres consists of the portion coming from the friction drag and that coming from the pressure drag. It may be of interest to observe the flow in which drag reduction phenomenon occurs around the rolling ball by the flow visualization technique.

Acknowledgment. The financial support of this work by a Grant-in-Aid for Scientific Research (No. 58550576) from the Ministry of Education, Science, and Culture is gratefully acknowledged.

\section{REFEREṄCES}

1. B. A. Toms, "Proceedings of the 1st International Congress on Rheology," Vol. II, North Holland, Amsterdam, 1949, p 135.

2. K. Imahara, K. Nakamura, T. Komatsu, and T. Nakagawa, Nippon Kagaku Kaishi, 1551 (1983).

3. P. D. Stein and H. N. Sabbah, Biorheology, 17, 301 (1980).

4. F. Yamashita, K. Nakamura, and T. Nakagawa, 


\section{Drag Reduction by Polystyrene}

Polym. J., 15, 563 (1983).

5: Y. Einaga, Y. Miyaki, and H. Fujita, J. Polym. Sci., Polym. Phys. Ed., 17, 2103 (1979).

6. H. Nagahora and H. Shimanouchi, Nihon Reoroji Gakkaishi, 14, 184 (1986).

7. R. M. Hubburd and G. C. Brown, Ind. Eng. Chem., Anal. Ed., 15, 212 (1943).
8. R. C. Little, R. L. Patterson, and R. Y. Ting, J. Chem. Eng. Data, 21, 281 (1977).

9. B. C. Ramakrishnan and F. Rodriguez, "Drag Reduction' in Polymers," Vol. 69, N. D. Sylvester, Ed., AIchE Symposium Series, 1973, p 52.

10. M. C. Kohn, J. Polym. Sci., Polym. Phys. Ed., 11, 2339 (1973). 